\title{
Death Due to Gunshot Injury; Is It a Case of Suicide or Homicide?
}

\author{
Ajay Kumar* \\ Assistant Director (Scene of Crime), FSL, India
}

Submission: November 29, 2017; Published: December 05, 2017

*Corresponding author: Ajay Kumar, Assistant Director (Scene of Crime), 0/o Inspector General of Police Hisar Range, Hisar, Haryana, India, Tel: 09996088880; Email: ajayadsoc@gmail.com

\begin{abstract}
One of the most important parts of safeguarding the scene of crime is to preserve the spot with least possible contamination and disturbance of physical clues. The first response to an incident should be prompt and meticulous. Evaluation of the spot of occurrence by the forensic experts leads to the determination of the type of incident to be investigated and the level of investigation to be conducted. Scene evaluation allows for the development of a plan for the coordinated identification, collection, and preservation of physical evidence and identification of witnesses. It also allows for the exchange of information among law enforcement personnel and the development of investigative strategies. In case of gunshot injury it is important to know the range of fire as well as handling of related physical evidences e.g. weapon (loaded/unloaded), cartridge, bullet, pellets, empty cases, gunshot residue (GSR) In this paper we will discuss about the processing of crime scene where a person was found dead in a cot with gunshot injury in his chest and then a fruitful reconstruction of the crime scene on the basis of physical evidences and their conditions and locations at the crime scene, nature of injuries etc. The matter was quite sensational and a case of murder (i.e. under section 302 of Indian penal code) was registered by the police against four persons on complaint of the family members of the deceased.
\end{abstract}

Keywords: Physical Evidence; Gunshot Residue; Gunshot Injury; Suicide; Homicide

Abbreviations: GSR: Gun Shot Residue

\section{Introduction}

On receipt of message from Police Station Bhuna, team of scientists of Forensic Science Laboratory of Haryana along with the investigating officer and concerned police officials visited scene of crime. Station house officer of Police Station, Bhuna who is the investigating officer of the case informed that dead body of a male person having gunshot injury in the chest was found lying in a cot in the agricultural fields of the village Bhundra of Dist. Fatehabad. The family members of the deceased alleged that he was shot dead by the persons whose name was written on the cuff of the shirt of the deceased. Town Bhuna came to a standstill because of agitations and processions for the demand of registration of a case of murder (i.e. under section 302 of Indian penal code) against the four persons and police finally registered a case of murder. Determination of suicide as a manner of death can be a complex process. Whenever gunshot injury is the cause of death, the evidences from the dead body are pivotal for determining whether it is a case of suicide or homicide depending upon the location of wound and range of fired determined from powder deposition. In addition to this comprehensive and methodical search of a crime scene ensures that all relevant evidences are detected and documented.A number of search methods is existing for an investigator to choose from to ascertain complete coverage and the most efficient use of resources. Crime scene documentation includes photography as an important part of a crime scene investigation with the primary purpose to provide the court with visual records of a crime scene, of the related evidence, and of the victims or suspects who were involved in the crime scene activity. Here another thing of immense importance is securing the crime scene i.e. to preserve the scene with least contamination and disturbance of physical evidence. Then the correct and welltimed evidence collection leads the proper reconstruction of a crime scene to establish how the crime has been committed and to the fruitful conclusion of the investigation. In the occurrences where fire arm is used, detection and identification gunshot residue (GSR) from fire arm discharge may provide valuable investigative information [1].

\section{Materials and Methods}

The crime scene was well preserved. Outward spiral method was used for the through search of scene of crime for the detection of all relevant physical evidences. All the physical evidences were located and documented with sketch as well as photographs. All required scientific methods and approaches were utilized during the handling forensic/physical evidences 
present on the spot and a proper reconstruction was made which involved scientific crime scene investigation, interpretation of patterned evidence at the scene, laboratory testing of the physical evidence, study of the injuries, systematic study of related case information and a logical hypothesis is developed.

\section{Scene of crime observations:}

a) It was observed that the dead body was lying with its facial side up in a cot placed near a tree in the open agricultural fields. The body was elongated in east- west direction as head in east direction and legs in west direction. Hands and lags of the deceased were lying straight. Eyes were open and mouth was partially closed. Rigor mortis was present in the body. Colour of the scalp hair, beard and mustaches was brownish and appeared as if dyed with henna. Eye brows of the deceased were noticed to be of black colour.

b) One brownish colour woolen shawl was found wrapped around the upper part of the body. When the shawl was removed blood was noticed on the cloths above the chest portion.

c) The deceased was wearing white kurta (shirt) payjama (trouser), cream half sleeve sweater, white vest and underwear having checks. Red sacred thread was observed around the left hand wrist. One lacerated wound with inverted margins of approx. size $3.5 \times 3.5 \mathrm{cms}$ was observed in the chest area i.e. approx. 2 inches below the left side nipple. Blackening was noticed around this wound. Corresponding holes were noticed on the vest, sweater and kameej (shirt) and blackening was present around these holes just above the wound. No exit wound could be noticed on the body of

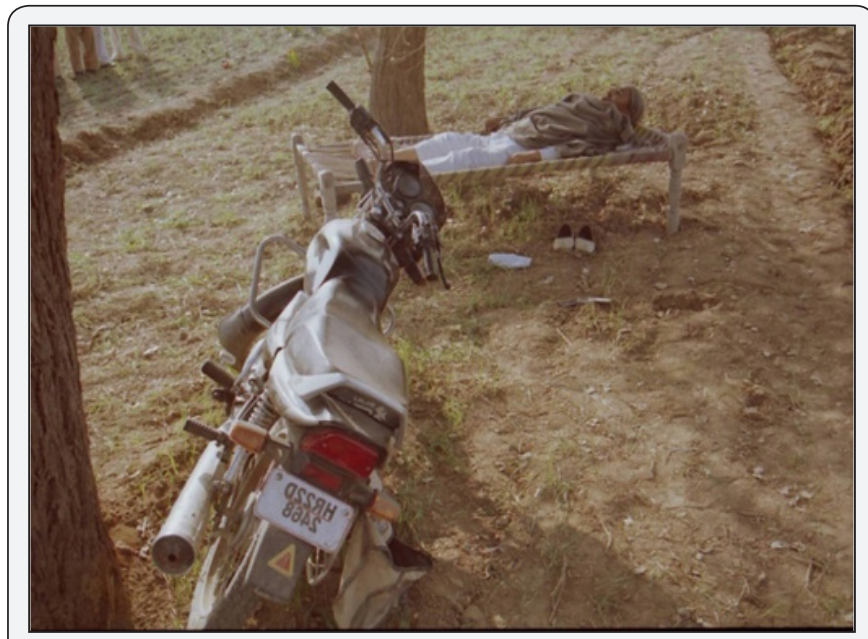

Figure 1: The view of the location of physical evidences detected on the spot. At circle 1- country made pistol, at circle 2- fired cartridge case of 12 bore, at circle 3- foot wear (Juti) of the deceased, at circle 4 - handkerchief, at circle 5- eye glasses, at point 6- motorcycle and at point 7- dead body of the deceased in a cot were detected. the deceased. It was noticed that blood traveled sideways of the chest and not down wards towards the legs. It appeared as if the bullet was struck his chest when he was in a posture lying straight on the cot and i.e. from the contact range as there was blackening present around the single entry wound and blasting effect was noticed over the corresponding holes in the cloths.

d) Three abrasions were noticed near the thumb of the left hand.

e) Small stains of blood were noticed on the payjama (trouser) over the right hip of the deceased.

f) One black and white colour ball point pen with black ink along with its cap was found under the dead body.

g) One country made pistol was found on the right side of the dead body at a distance of approx. 4 feet from the cot. One cartridge case of caliber 12 gauge/bore (K F 12) was present inside the barrel of the pistol.

h) One same kind of cartridge case was noticed at distance approx 7.5 feet away in east direction from the cot. A pair of footwear (juti) was noticed to be placed properly under the cot. White handkerchief was found lying near the juti. One white colour mobile set (Nokia 1100), a diary, key of the motorcycle and spectacles with photocromatic glasses were found in the pocket of the shirt (kurta) of the deceased.

i) One black colour motor cycle (Bajaj CT 100) bearing Reg. No. HR 22 D 2468 was observed to be parked near the tree at a distance approx. 8 feet away in the north direction from the cot. The motorcycles was intact and in perfect working order (Figures 1-10).

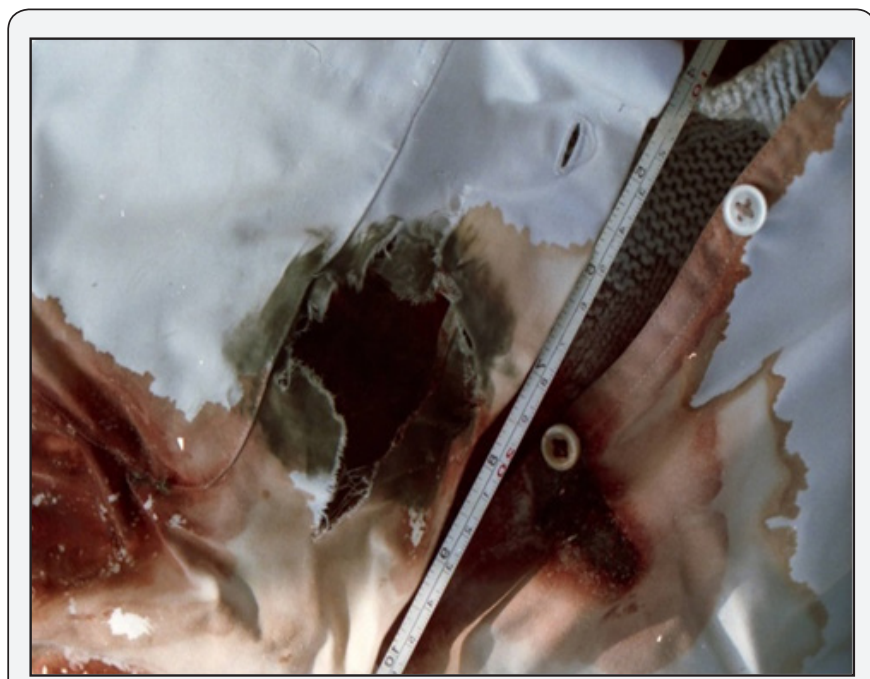

Figure 2 : The view of the huge deposition of blackening and blood around the hole in the white shirt of the deceased. The surface around the hole was irregular and ragged and had blasting effect due to expansion of gases which indicate the contact fire. 


\section{Journal of Forensic Sciences \& Criminal Investigation}

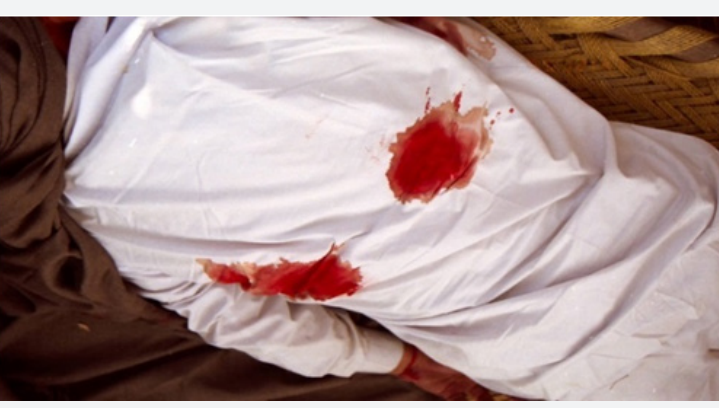

Figure 3 : The back side conditions of the deceased which shows blood stain on the both side of the back as blood travelled down to the back from the left and right side of the wound in the chest of the deceased which indicated that the deceased was in lying position not in the standing or sitting position when received the bullet injury.

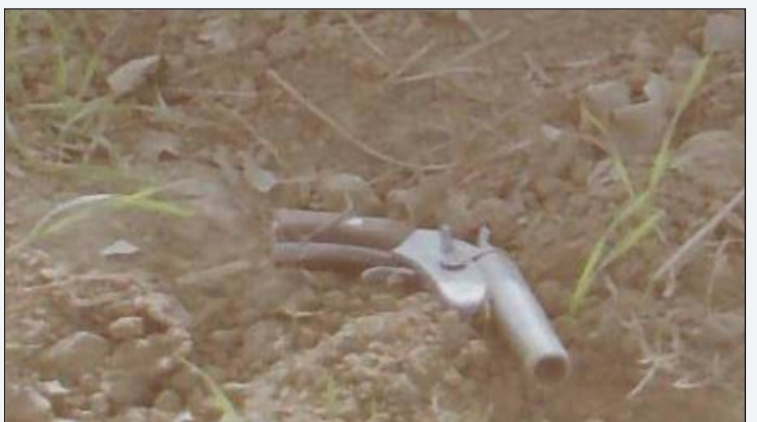

Figure 4 : The country made pistol of 12 bore/caliber detected on the spot as mentioned in figure 1 .

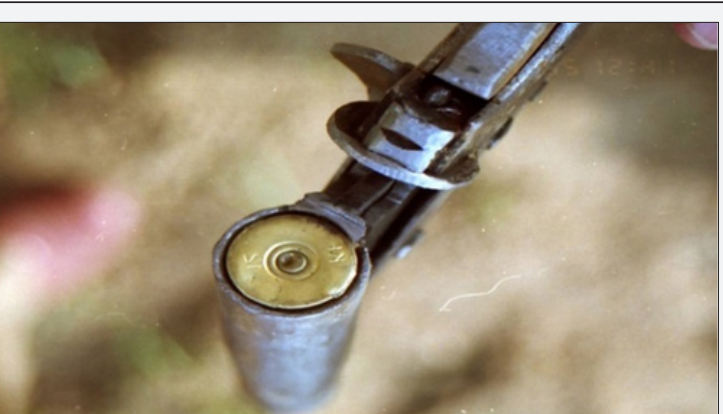

Figure 5 : The fired cartridge (KF 12) case inside the barrel of country made pistol.

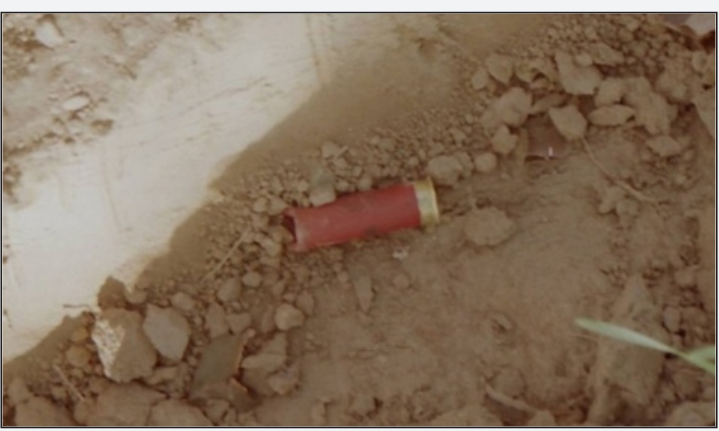

Figure 6 : The fired cartridge (KF 12) case i.e. similar as shown in figure 5 which was observed to be lying on the spot of occurrence.

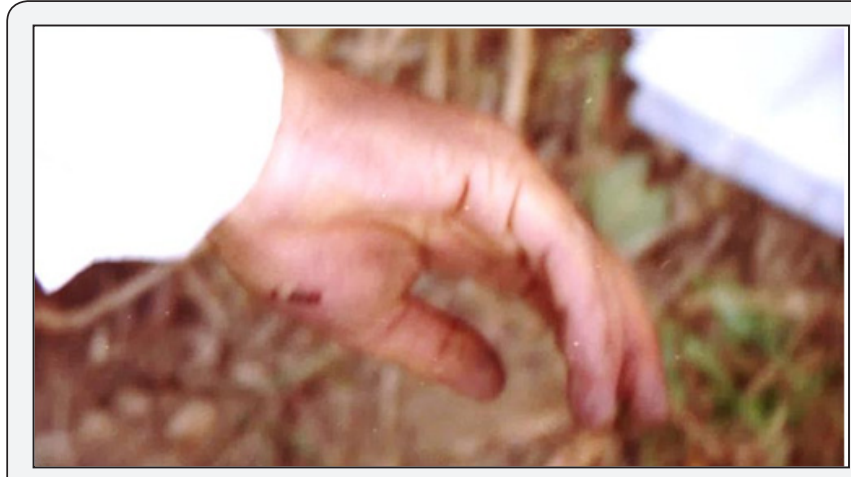

Figure 7 : The abrasions with blood stain near the thumb of the left hand.

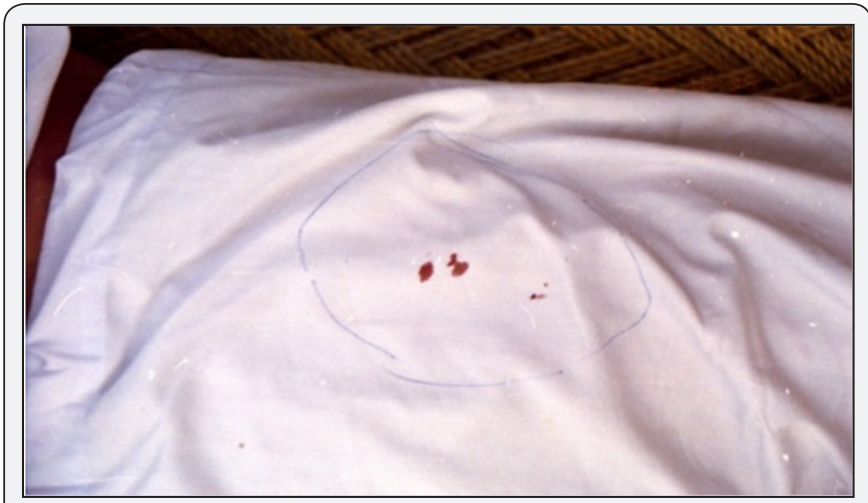

Figure 8 : The small stains of blood on the pyjama (trouser) over the right hip area.

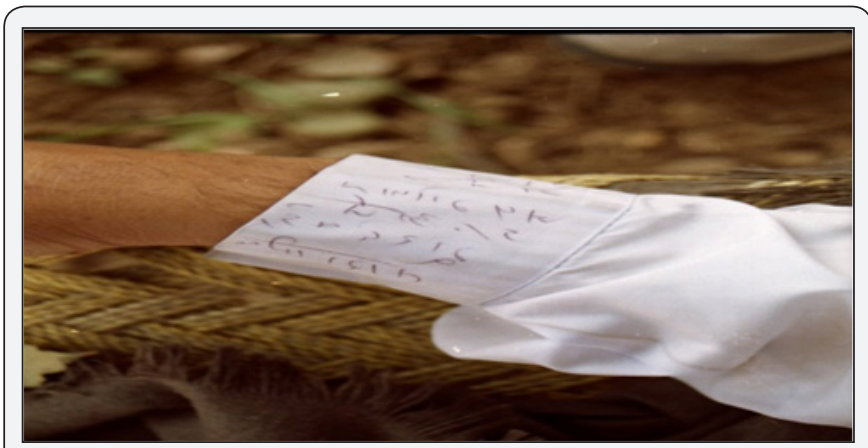

Figure 9 : The names written with black ink on the cuff of.

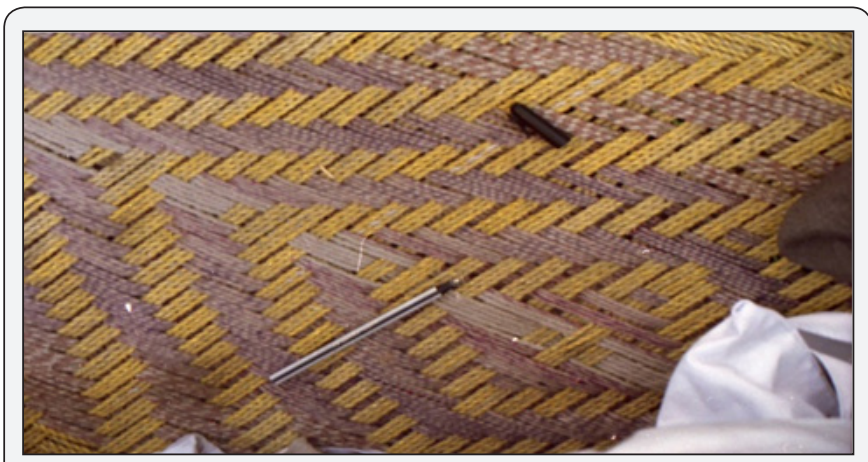

Figure 10 :The black ball point pen along with its cap lying on the cot. 
j) From the observations of the dead body and scene of crime, following inferences are drawn: The spot was lonely place. No any struggle took place at the SOC as no such evidence could be observed at the spot and dead body. The victim was shot at from the contact range as heavy deposition of shoot particles seen around the entry wound. The victim received gunshot in lying position because blood stain pattern suggested this and the blood stain pattern also ruled out the transportation of the dead body. Two cartridges were fired at from the country made pistol because physical evidence of two fires were found at the spot of occurrence. The victim came to the spot by a bike found parked on the scene and the key of the bike was found in the pocket of victim's shirt [2].

\section{Observations from the post mortem examination of the deceased:}

On lacerated wound $1.5 \times 1.5$ on front chest below and medial to left nipple $(5.5 \mathrm{cms})$ margins inverted with blackening around, bleeding from the wound present. On dissection underlying ribs are fractured, wound is vertical. Bone pieces, clothes pieces, red colored and pellets are present. Multiple pellets and red colored plastic structure are taken out.

Inferences drawn on the basis of autopsy examination: The deceased received the gunshot from contact range. The absence of any other injuries except the only mentioned gunshot injury suggested no any kind of struggle with the deceased [3].

The ballistic analysis: During the autopsy multiple pellets and red coloured plastic structure taken for ballistic examination purposes. These Multiple pellets and red coloured plastic structure along with the cotton swabs of both hand of deceased, the two cartridge cases and the pistol were sent to the ballistic division of laboratory and analysed.

a) Inferences drawn on the basis of ballistic examination in the forensic science lab: Products of combustion of smokeless powder were detected from the barrel of country made pistol chambered for 12-bore cartridges. Test firings were done in the laboratory from pistol marked. Its firing mechanism was found in working order.

b) The country made pistol (chambered for 12-bore cartridges) is a firearm as defined in Arms Act 54 of 1959. Its firing mechanism was found in working order.

c) Both of the 12-bore fired cartridge cases have been fired from country made pistol and not form any other firearm even of the same make and bore, because every firearm has got its own individual characteristic marks.

d) The pellets were found to be size (B). The plastic air cushion wad is normally loaded in shotgun cartridges including 12 bore.

e) Gunshot residue was detected from both the cotton swab contained in the swabs of both hands of the deceased.
The handwriting analysis: black ball pen, cuff of sleeve of the deceased's shirt on which names written with black ink along with the authenticated handwriting sample were sent to document division of forensic science lab for analysis/ comparison [4].

Inferences drawn from the analysis: There is no divergences observed between standard (deceased's authenticated handwriting) and questioned (handwriting on the cuff of the sleeve of the shirt) writings and the aforesaid similarities in writing habits are significant and sufficient and can't be attributed to accidental coincidence and in combination with the surrounding conditions as well as condition of the writer, leads to the opinion that all the handwriting on the cuff of the sleeve of the shirt and deceased's authenticated handwriting sample have been written by one and the same person. The writings on the cuff of the sleeve of the deceased shirt have been written with black ball point pen ink found on the spot which was containing refill of black ball point pen ink and tint of ink of ball pen appears to be similar to the tint of ink writings marked [5].

\section{Discussion}

In situations when a shotgun is discharged into the chest during a contact (loose or hard) or close range shot (up to about $15-30 \mathrm{~cm}$ or $6-12$ inches), the pellets contained in the cartridge have not had time to spread, and they enter the body as a single mass. The appearance of gunshot entrance wounds on the body depends upon many factors including, the type of firearm, the type of ammunition, the location of the wound on the body, and the circumstances of how a wound was sustained. Firearm wounds to the chest invariably have typical entry and exit wounds or solely entry wounds when the projectile has not ejected the body. Usually, distinctive features present on skin and bone are available to distinguish between these wounds. Ballistics is however an intriguing science. Multiple factors like the type of ammunition used, muzzle velocity and target characteristics play a role in the final outcome of a gunshot. In situations involving actual or suspected use of firearms, detection and identification of residual materials from firearms discharge may provide valuable investigative information. These residues are principally composed of burnt and un burnt particles from the propulsive charge, as well as components from the primer, the bullet, the cartridge case and the firearm itself, and they are generally called gunshot residue (GSR). Detection and identification of GSR are commonly performed with the aim of determining whether or not a suspect fired a gun, to determine a bullet entrance hole or to estimate a firing distance [6,7].

\section{Conclusion}

No mark of struggle was detected from the spot as well as from the dead body indicating no force has been used against the deceased. The presence of bike of the deceased on spot, its keys with deceased suggested that the deceased came to the spot by his bike and he parked the bike and kept its keys with 
him. No blood stains could be observed from the spot except on dead body and the cot on which it was lying which ruled out the transportation of the dead body [8]. Two empty cartridges were found at spot i.e. one inside the pistol and another on the spot indicating two fires were made from the pistol. Blood pattern over the dead body suggested that the deceased received bullet in lying conditions.

Shoot pattern over the wound suggested the contact range fire. Presence of gunshot residue (GSR) over the palms of the deceased indicated that the deceased made fire. The writings on the cuff of the sleeve of the deceased shirt have been written with black ball point pen ink found on the spot and this handwriting matched with the deceased's authenticated handwriting sample hence indicating the deceased written the names on the cuff of his shirt. Hence, it was concluded that deceased came to the spot of incident by his bike and written names on the cuff of sleeve of his shirt and then he removed the empty cartridge from the pistol, then reloaded and shot himself in the chest from contact range, hence it was a case of suicide.

\section{References}

1. Clark SC (1999) Death Investigation: A Guide for the Scene Investigator. National Institute of Justice, Washington, USA.

2. Cassidy M (2000) Smooth-bore firearm injuries Chapter 5 In: Mason JK and Purdue BN (Eds,) The Pathology of Trauma ( $3^{\text {rd }}$ edn.), Arnold Publishers, London, UK.

3. Breitenecker R (1969) Shotgun wound patterns. American Journal of Clinical Pathology 52(3): 258-269.

4. Di Maio VJM (1999) Gunshot Wounds: Practical Aspects of Firearms, Ballistics and Forensic Techniques ( $2^{\text {nd }}$ edn). CRC Press LLC, Florida, USA.

5. Cullen EF, Luckasevic TM (2010) Suicide with a homemade shotgun: case report and review of literature. Am J Forensic Med Pathol 31(3): 255-257.

6. Francesco SR, Pierre M (2001) Identification of gunshot residue: a critical review. Forensic Science International 119(2): 195-211.

7. Karl GS, Beat PK (1994) Wound Ballistics and the Scientific Background. Elsevier publishers, Netherlands.

8. K Sellier (1991) Shot range determination In: A maehley, RL Williams (Eds.), Forensic Science Progress, Springer, Berlin.

Your next submission with Juniper Publishers
will reach you the below assets
- Quality Editorial service
- Swift Peer Review
- Reprints availability
- E-prints Service
- Manuscript Podcast for convenient understanding
- Global attainment for your research
- Manuscript accessibility in different formats
( Pdf, E-pub, Full Text, Audio)
- Unceasing customer service
Track the below URL for one-step submission
https://juniperpublishers.com/online-submission.php -

